\title{
Impairment of sterol biosynthesis leads to phosphorus and calcium accumulation in Leishmania acidocalcisomes
}

\author{
Marcos A. Vannier-Santos, ${ }^{1}$ Andrea Martiny, ${ }^{1}$ Ulysses Lins, ${ }^{2}$ \\ Julio A. Urbina, ${ }^{3}$ Valéria M. Borges ${ }^{4}$ and Wanderley de Souza ${ }^{4}$
}

Author for correspondence: Marcos A. Vannier-Santos. Tel: +55 21260 6963. Fax: +55 212808193. e-mail:vannier@biof.ufrj.br

1 Laboratório de Biologia Celular Parasitária, Programa de Biologia Celular e Parasitologia Instituto de Biofísica Carlos Chagas Filho, Universidade Federal do Rio de Janeiro, CCS, Bloco G, Ilha do Fundão, Rio de Janeiro, 21949-900, Brazil

2 Setor de Microscopia Eletrônica e Departamento de Microbiologia Geral, Instituto de Microbiologia Professor Paulo de Góes, Universidade Federal do Rio de Janeiro, Brazil

3 Laboratório de Química Biológica, Instituto Venezoelano de Investigaciones CientíficasIVIC, Venezuela

4 Laboratório de Ultraestrutura Celular Hertha Meyer, Instituto de Biofísica Carlos Chagas Filho, Universidade Federal do Rio de Janeiro, Brazil
The induction of the formation of inclusion vesicles in Leishmania amazonensis by the sterol biosynthesis inhibitors (SBI) ketoconazole and terbinafine has been reported previously. These compartments were recently identified as acidocalcisomes. By the use of electron spectroscopic imaging and energy loss spectroscopy, the presence of calcium, phosphorus and oxygen in the electron-dense inclusions located within the acidocalcisomes has been demonstrated. Endoplasmic reticulum cisternae formed membrane whorls which enclosed large portions of the cytoplasm and sometimes circumscribed acidocalcisomes. In addition, acid phosphatase activity, as well as the endocytic tracers horseradish peroxidase and gold-labelled transferrin and cystatin C were detected within these organelles in both SBI-treated and untreated parasites. These data suggest that impairment of sterol biosynthesis induces the biogenesis of acidocalcisomes and triggers an autophagic process that leads to intersection of the endosomal/lysosomal system with the acidocalcisomes.

Keywords: sterol biosynthesis inhibitors, ketoconazole, terbinafine, acidocalcisome, Leishmania

\section{INTRODUCTION}

The antifungal sterol biosynthesis inhibitors (SBI) present microbicidal activity against trypanosomatid parasites both in vitro and in vivo (Behamdan et al., 1997; Berger \& Fairlamb, 1992; Berman, 1988; Chance, 1995; Olliaro \& Bryceson, 1993; Rangel et al., 1996; Urbina et al., 1996; Vannier-Santos et al., 1995; Werbovetz et al., 1992). SBI such as ketoconazole and terbinafine have additive or synergistic leishmanicidal effects (Vannier-Santos et al., 1995) and terbinafine was shown to revert the azole resistance in Leishmania braziliensis (Rangel et al., 1996). We have previously noticed (Vannier-Santos et al., 1995) that these SBIs induce, among other alterations, the enhanced appear-

Abbreviations: ER, endoplasmic reticulum; ESI, electron spectroscopic imaging; SBI, sterol biosynthesis inhibitors. ance of inclusion vesicles and therefore may constitute useful tools for studying the cell biology of this ubiquitous but obscure organelle in trypanosomatid parasites.

Inclusion vesicles were previously described as membrane-bound organelles displaying electron-dense cores associated with the luminal face of the membrane (Bird et al., 1966; Vickerman \& Preston, 1976; Vickerman \& Tetley, 1977). This organelle received different designations such as electron-dense granules (Carvalho et al., 1979) and lysosomes (Macadam \& Williamson, 1974a, b; Slomianny \& Prensier, 1990; Williamson \& McLaren, 1981) based upon their ultrastructural appearance and the presence of acid phosphatase activity as reported in Trypanosoma gambiense (Seed et al., 1967), Trypanosoma rhodesiense (Dvorak et al., 1988) and Herpetomonas samuelpessoai (Carvalho et al., 1979). 


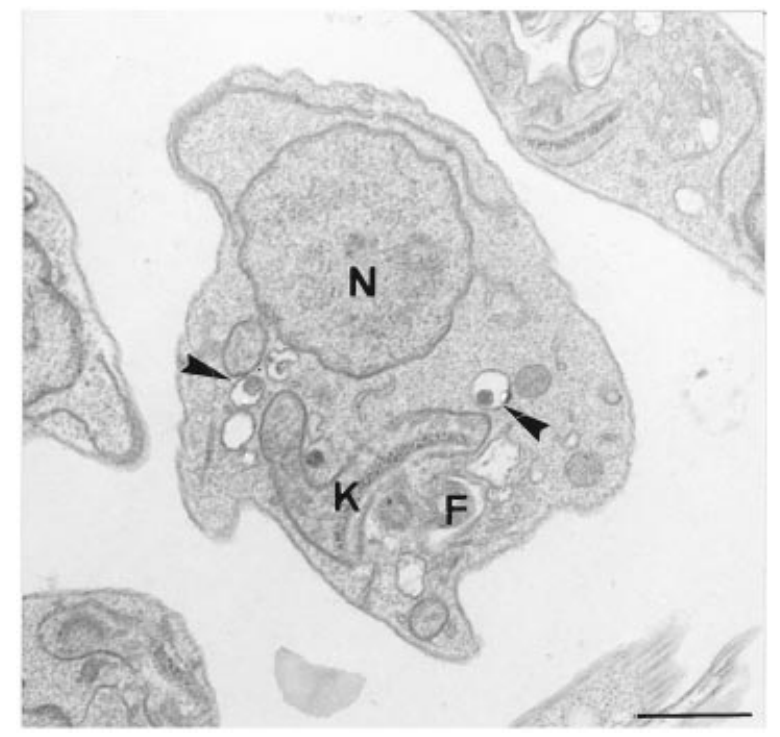

Fig. 1. General view of an untreated $L$. amazonensis promastigote, presenting normal appearance of the cell with nucleus $(N)$, kinetoplast $(K)$ and the emerging flagellum $(F)$. Acidocalcisomes (arrowheads) are usually scarce in these cells. Bar, $1 \mu \mathrm{m}$.

Understanding the physiological role of these so-called inclusion vesicles has been hampered by the difficulty in matching early histochemical data with current electron microscopy observations, particularly whenever techniques such as quick freezing, freeze-substitution and ultracryomicrotomy are employed (Dvorak et al., 1988; Scott et al., 1997). Thus, organelles of distinct origins and chemical composition may be mistaken (Vickerman \& Preston, 1976; Herbert, 1965a, b) and the identification of this compartment on electron micrographs has been considered purely arbitrary (Watson \& Lee, 1975). Despite its widespread distribution in diverse organisms, little is known about the functional dynamics of these structures, particularly in protozoa, and most of the knowledge in this field relies on early publications. Similar organelles in $H$. samuelpessoai were suggested to comprise endosomal/lysosomal compartments based upon the presence of acid phosphatase activity and incorporation of iron as assessed by X-ray spectra (Carvalho \& De Souza, 1977), in addition to the presence of a porphyrin prosthetic group peroxidase activity (Carvalho et al., 1979). Similarly, they were termed pigment bodies in Trypanosoma cyclops and may correspond to sites of intracellular accumulation of haemoglobin ingested by endocytosis (Heywood et al., 1974; Weinman, 1971). Vickerman \& Tetley (1977) suggested that inclusion vesicles and pigment bodies, detected by both light and electron microscopy (Heywood et al., 1974), are different entities, but the possibility that they comprise distinct stages of a single compartment was not excluded. It must be noted that the former is more frequent in both $T$. cyclops (Heywood et al., 1974) and H. samuelpessoai (Carvalho
\& De Souza, 1977) cultivated with haemoglobin and haemin, respectively. Similar electron-dense membranebound vesicles were regarded as stored metabolic products in Herpetomonas megaseliae (Janovy et al., 1974). More recently (Scott \& Docampo, 1998; Scott et al., 1997), structurally identical compartments in Trypanosoma cruzi were ascribed to be the acidocalcisomes (Vercesi et al., 1994).

In this study we report data on the formation and composition of acidocalcisomes in ketoconazoleand terbinafine-treated and untreated Leishmania amazonensis promastigotes. The possible intersection of this organelle with the endosomal/lysosomal system is suggested.

\section{METHODS}

Parasites. The MHOM/Josefa/75/Br strain of Leishmania amazonensis (kindly provided by $\mathrm{Dr} \mathrm{C}$. A. Cuba-Cuba, Universidade de Brasília, Brazil) was isolated from a human case of diffuse cutaneous leishmaniasis. It was maintained by hamster footpad inoculation and in axenic culture in Warren medium supplemented with $10 \%$ foetal calf serum at $26{ }^{\circ} \mathrm{C}$.

Ultrastructure and biogenesis of acidocalcisomes. Promastigotes and amastigotes were incubated with different concentrations of either terbinafine or ketoconazole $(0 \cdot 001-1 \mu \mathrm{M})$ in Warren medium, fixed in $2.5 \%$ glutaraldehyde and $0.2 \%$ picric acid in $0 \cdot 1 \mathrm{M}$ sodium cacodylate buffer, $\mathrm{pH} 7 \cdot 2$, after 72 and 96-h treatments. Cells were washed, post-fixed in $1 \%$ $\mathrm{OsO}_{4}, 0.8 \%$ potassium ferricyanide in the same buffer, dehydrated in acetone and embedded in Epon. Thin sections were collected on 400 mesh copper grids, stained with uranyl acetate and lead citrate and observed with a Zeiss CEM 902 transmission electron microscope.

Endosomal/lysosomal origin of acidocalcisomes. Treated and untreated parasites were incubated for 60-120 min in the presence of horseradish peroxidase (Graham \& Karnovsky, 1966) or $10 \mathrm{~nm}$ gold-labelled human transferrin as described by Borges et al. (1998). Purified human cystatin C (kindly provided by Dr Julio Scharfstein, Universidade Federal do Rio de Janeiro, Brazil) was conjugated to $10 \mathrm{~nm}$ gold particles as described by Roth (1983). For acid phosphatase activity detection, treated and untreated parasites were washed in PBS, $\mathrm{pH} 7 \cdot 2$, briefly fixed in cold $1 \%$ Grade I glutaraldehyde in $0 \cdot 1 \mathrm{M}$ cacodylate buffer, $\mathrm{pH} 7 \cdot 2$, rinsed in $10 \mathrm{mM}$ sodium acetate buffer, $\mathrm{pH} 5 \cdot 0$, at $4{ }^{\circ} \mathrm{C}$ and incubated for $45 \mathrm{~min}$ at $37^{\circ} \mathrm{C}$ in the same buffer containing $1 \mathrm{mM} \mathrm{CeCl}, 1 \mathrm{mM}$ sodium- $\beta$-glycerophosphate, $5 \%$ sucrose (Robinson \& Karnovsky, 1983). Cells were then washed, fixed again in $2.5 \%$ glutaraldehyde in cacodylate buffer for $1 \mathrm{~h}$ at room temperature and processed as described above. Sections were stained with lead citrate and observed under a Zeiss 900 electron microscope.

Composition of the acidocalcisomes. Electron spectroscopic imaging (ESI) of acidocalcisomes was carried out in ultrathin sections $(30-50 \mathrm{~nm})$ of treated specimens collected on 400 mesh nickel grids. The unstained material was analysed on a Zeiss CEM 902 electron microscope equipped with an integrated magnetic prism and attached to a digital image analysis system (IBAS, Kontron). The microscope was operated in image mode with an energy selecting slit aperture of $20 \mathrm{eV}$. The accelerating voltage was $80 \mathrm{kV}$ and the 

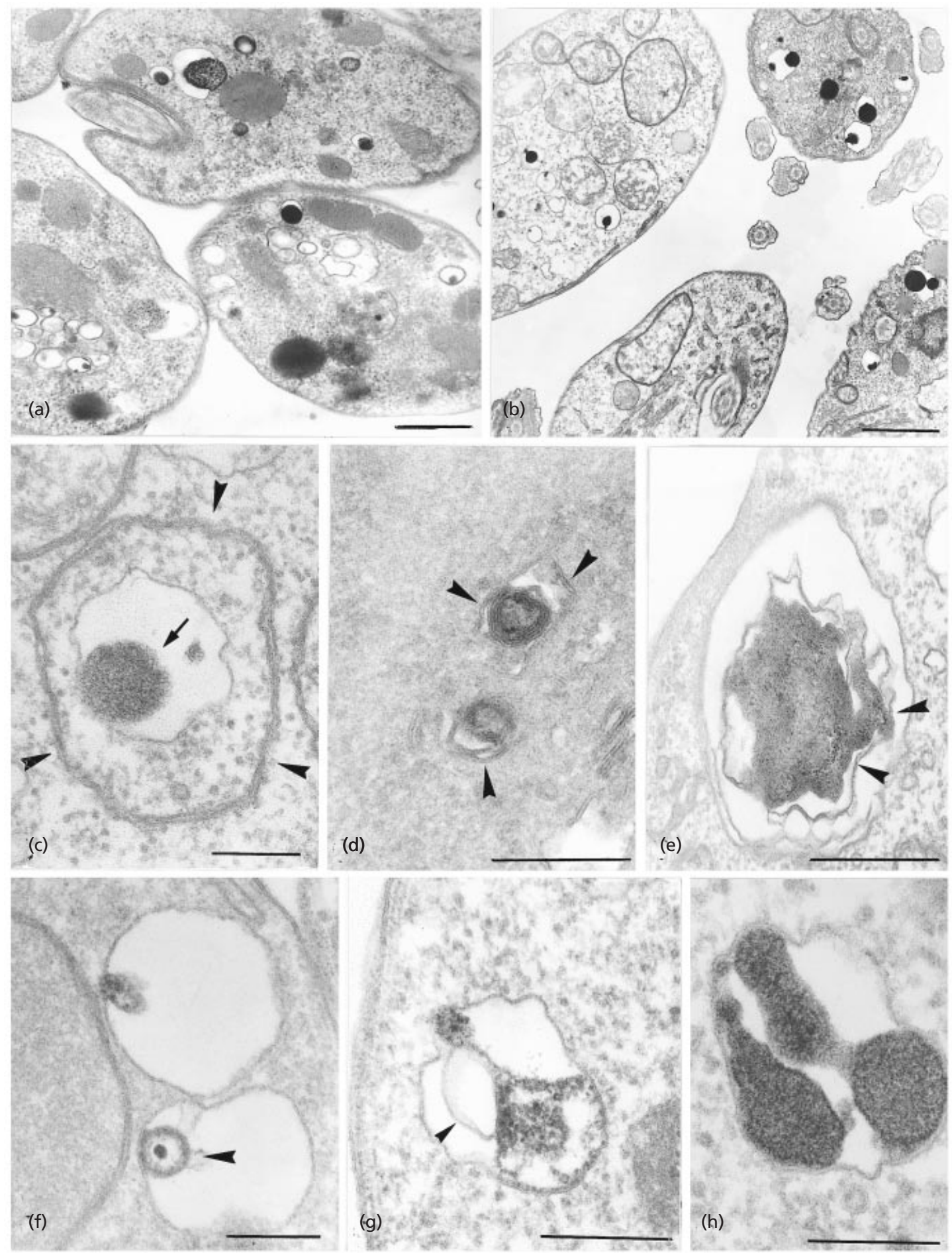

Fig. 2. Effects of the SBI ketoconazole $(1 \mu \mathrm{M})$ and terbinafine $(10 \mu \mathrm{M})$ on $L$. amazonensis parasites after a $4 \mathrm{~d}$ treatment. (a, b) Overview of ketoconazole- (a) and terbinafine-treated (b) promastigotes, displaying an increased number and volume of acidocalcisomes. (c) Circular ER cisterna enclosing a portion of the cytoplasm (arrowheads) and an acidocalcisome (arrow). (d) SBI-treated parasite showing vesicles containing membrane whorls (arrowheads). (e) Vacuole presenting a myelin-like figure. Note that membrane units are visible solely at the periphery (arrowheads). (f) Vesicles presenting acidocalcisome-like cores with a heterogeneous appearance. Concentric patterns are particularly frequent (arrowhead). (g) Acidocalcisome-like compartment presenting electron-dense, amorphous material associated with membrane units (arrowhead). (h) A bizarrely shaped acidocalcisome in an SBI-treated parasite. Bars, $1 \mu \mathrm{m}$ (a, b) and $0.25 \mu \mathrm{m}(\mathrm{c}-\mathrm{h})$. 


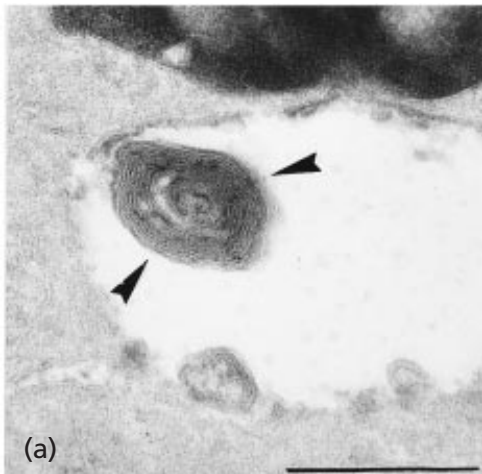

(a)

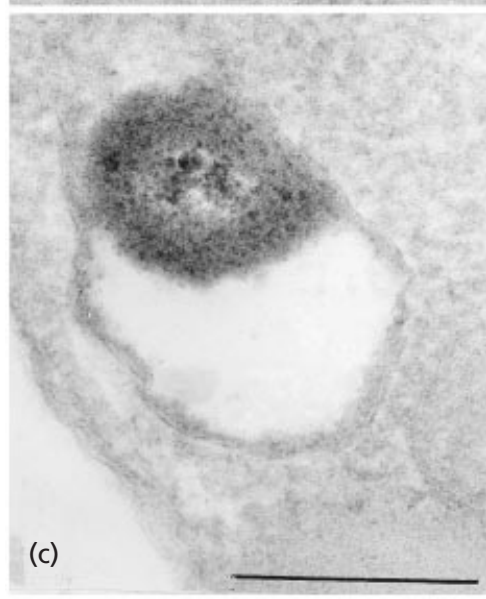

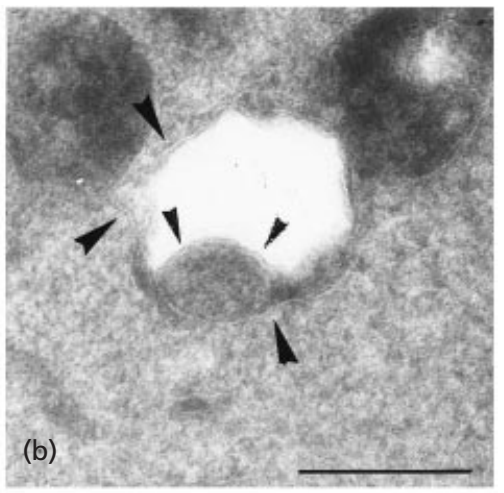

(d)

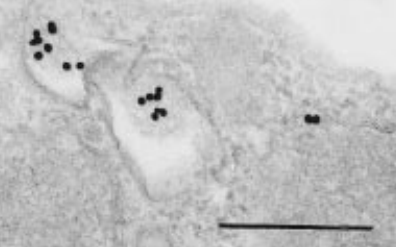

(e)
Fig. 3. Endosomal/lysosomal nature of acidocalcisomes in terbinafine-treated and untreated Leishmania. (a) Detection of acid phosphatase activity in vesicle containing a myelin-like structure (arrowheads). (b) Acidphosphatase-positive acidocalcisome. It can be observed that the organelle core is limited by a membrane (arrowheads). (c-e) Detection of ingested horseradish peroxidase (c), gold-labelled transferrin (d) and gold-labelled cystatin C (e) within inclusion vesicles or acidocalcisomes. Bars, $0.25 \mu \mathrm{m}$. condenser and objective apertures were 100 and $90 \mu \mathrm{m}$, respectively. Elemental distribution images of oxygen and phosphorus were calculated using the three windows method (Reimer, 1991). This method uses two images above and one below the absorption edge of an element to remove background electrons and obtain a distribution map of that element. For oxygen, the energy windows below the edge were centred at 490 and $515 \mathrm{eV}$ and the one above centred at $545 \mathrm{eV}$. For phosphorus, the two energy windows below were 100 and $110 \mathrm{eV}$ and the one above was $150 \mathrm{eV}$. For calcium, the two-window method was used with an energy window below the edge at $330 \mathrm{eV}$ and another above the edge at $360 \mathrm{eV}$. Elemental maps were calculated by digital subtraction of these two images. For electron energy loss spectroscopy (EELS), spectra were recorded by an integrated photomultiplier operated by dedicated software via an IEEE 488 interface (Kontron). The microscope was operated in spectrum mode at $80 \mathrm{kV}$ using an objective aperture of $30 \mu \mathrm{m}$. The energy selecting slit aperture was approximately $2 \mathrm{eV}$. Intensities of energy losses during acquisition were measured at each $1 \mathrm{eV}$ by a digital multimeter.

Drugs. Terbinafine [SF-86327; (E)-N-(6,6-dimethyl-2-hepten4-ynyl)-N-methyl-1-naphthalene methanamine] was provided by A. Lindenmann and H. Stähelin (Sandoz, Switzerland) through Luís Rodrigues (Sandoz, Venezuela). It was added in cultures as a DMSO solution. The final DMSO concentrations never exceeded $1 \%(\mathrm{v} / \mathrm{v})$ and had no effect on the proliferation or morphology of parasites. John Russe (Janssen Pharmaceutica, Caracas, Venezuela) provided ketoconazole \{cis-1-acetyl-4-[4-([2-(2,4-dichlorophenyl)-2- (1H-imidazol1-ylmethyl)-1,3-dioxolan-4-yl]-methoxy)phenyl]piperazine\}. The drug was added as an aqueous solution titrated to $\mathrm{pH} 2 \cdot 4$ with $\mathrm{HCl}$ and sterilized by filtration.

\section{RESULTS}

\section{Morphological alterations induced by inhibition of sterol biosynthesis}

The general appearance of a control promastigote displaying inclusion vesicles or acidocalcisomes is shown in Fig. 1. Ketoconazole (Fig. 2a) and terbinafine (Fig. 2b) induced the formation of numerous and diverse acidocalcisomes in both developmental forms of the parasite compared to control cells. Many SBI-treated cells presented circular endoplasmic reticulum (ER) cisternae enveloping portions of the cytoplasm. Acidocalcisome-like organelles could be found in association with such ER profiles (Fig. 2c). SBI-treated parasites often displayed membranous whorls within vacuoles (Fig. $2 \mathrm{~d}, \mathrm{e}$ ) and in the larger ones the membrane units were not always visible at the centre of the myelinlike structures (Fig. 2e). These membrane-containing compartments intergraded with acidocalcisomes, which initially presented heterogeneous cores, often showing centre-periphery differences in electron density and granularity (Fig. 2f). Polymorphic, membrane-containing concretions (Fig. $2 \mathrm{~g}$ ) apparently gave rise to bizarrely shaped acidocalcisomes (Fig. 2h).

\section{Endosomal/lysosomal origin of acidocalcisomes}

Acid phosphatase cytochemical detection assays were performed to investigate the possible relation between acidocalcisomes and lysosomal compartments. Acid 

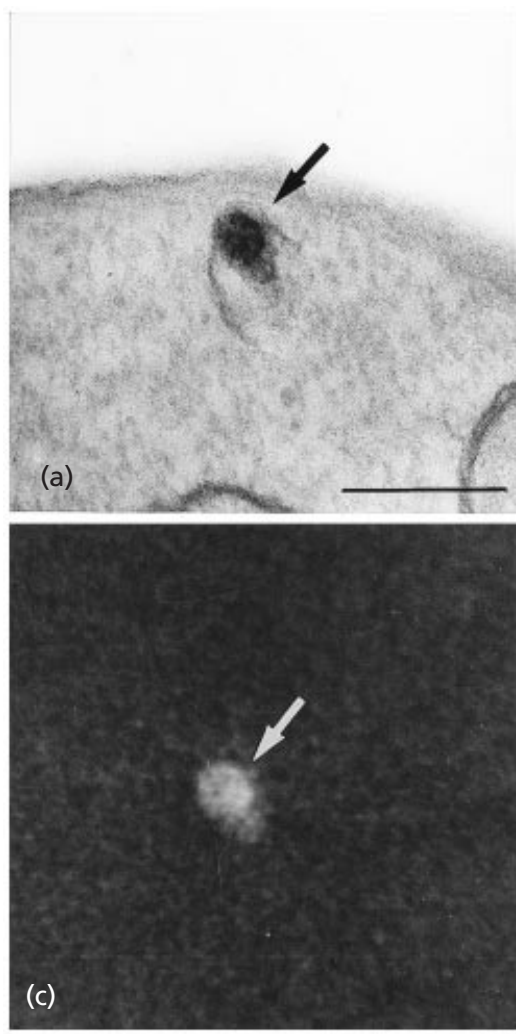
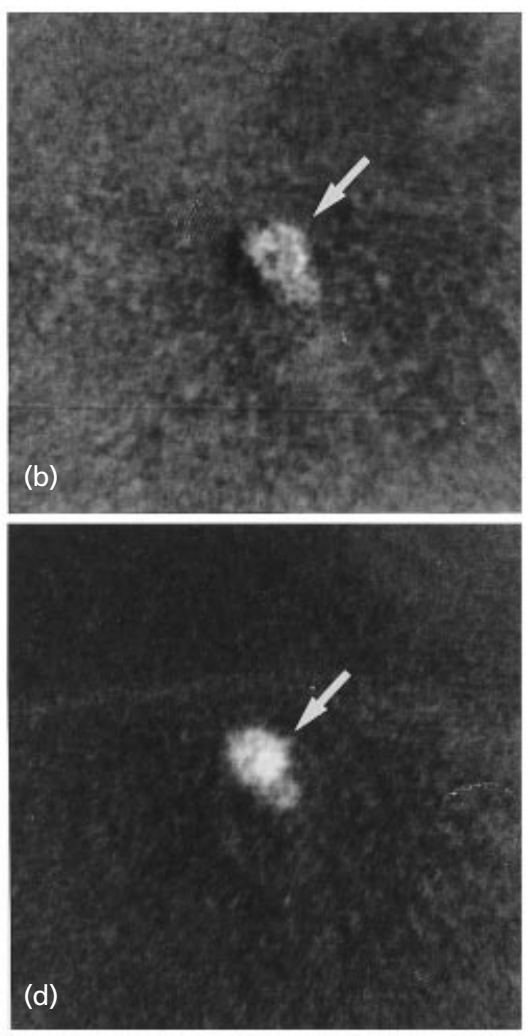

Fig. 4. ESI (b-d) of an L. amazonensis acidocalcisome shown in (a). Promastigotes were incubated with $10 \mu \mathrm{M}$ terbinafine for $3 \mathrm{~d}$ and processed for transmission electron microscopy as described in Methods. The phosphorus elemental map (b) was calculated by the three-window method using two energies below (100 and $110 \mathrm{eV}$ ) and one above $(155 \mathrm{eV})$ the energy edge $(132 \mathrm{eV})$. The oxygen elemental map (c) was also calculated by the three-window method using two energy windows below (490 and $515 \mathrm{eV})$ and one above ( $545 \mathrm{eV})$ the energy edge $(532 \mathrm{eV})$. The calcium elemental map (d) was calculated by the two-window method using one energy window above $(360 \mathrm{eV})$ and one below (330 eV) the energy edge $(346 \mathrm{eV})$. A homogeneous distribution of the three elements can be observed within the acidocalcisome. Bar, $0.5 \mu \mathrm{m}$. phosphatase activity was observed both in compartments containing myelin-like figures (Fig. 3a) and acidocalcisomes (Fig. 3b) in SBI-treated promastigotes. It is noteworthy that the organelle core was membranebound (Fig. 3b). Such membrane units were not observed in routinely prepared samples.

We performed assays in the presence of the endocytic tracers horseradish peroxidase (fluid-phase endocytosis, Fig. 3c), gold-labelled transferrin (receptor-mediated endocytosis, Fig. 3d) and gold-labelled cystatin C (Fig. $3 \mathrm{e}$ ) with SBI-treated and untreated parasites to verify the possible correlation of acidocalcisomes with endosomes. The three tracers were observed in inclusion-containing compartments.

\section{Phosphorus, oxygen and calcium content of acidocalcisomes}

Inelastically scattered electrons with element-specific energy losses were used to determine the distribution of several elements in promastigotes before (not shown) and after terbinafine treatment. Both the electron energy loss spectra and elemental maps of the acidocalcisomes revealed the presence of phosphorus, oxygen and calcium. Elemental mapping of acidocalcisomes showed a homogeneous distribution of phosphorus (Fig. 4b), calcium (Fig. 4c) and oxygen (Fig. 4d) all over the acidocalcisome cores (Fig. 4a). Electron energy loss representative spectra confirmed the presence of these elements by demonstrating the calcium $\mathrm{L}_{2,3}(346 \mathrm{eV})$, oxygen $\mathrm{K}(532 \mathrm{eV})$, nitrogen $(402 \mathrm{eV})$ edges (Fig. 5a). The $\mathrm{L}_{2,3}$ edge for phosphorus (132 eV; Fig. 5b) and occasionally zinc (not shown) were also detected in this organelle. We cannot exclude the possibility that other elements were washed out during sample processing and/or sectioning.

\section{DISCUSSION}

The observations reported show that incubation of Leishmania in the presence of drugs that inhibit the biosynthesis of sterols led to the appearance of increased numbers of polymorphic structures previously recognized as volutin granules and inclusion vesicles (Vickerman \& Preston, 1976) and more recently characterized as acidocalcisomes (Scott et al., 1997). The organelles observed here presumably comprise acidocalcisomes since they are the only compartments that accumulate significant amounts of calcium. It has been shown that the electron-dense inclusions found within this organelle in T. cyclops (Heywood et al., 1974), T. rhodesiense (Macadam \& Williamson, 1974a, b) and $T$. cruzi (Carvalho et al., 1979) contain phosphorus and calcium. These observations were confirmed in this study by ESI and energy loss spectroscopy of acidocalcisomes found in control and SBI-treated $L$. amazonensis. In addition, oxygen was also detected, reinforcing the presumed presence of oxygenated phosphorus compounds. Recent studies using ${ }^{31} \mathrm{P}$ NMR have shown that this compound is inorganic pyrophosphate (Urbina et al., 1998).

Studies carried out in T. cruzi have shown the presence of a vacuolar-type $\mathrm{H}^{+}$-ATPase (Scott \& Docampo, 


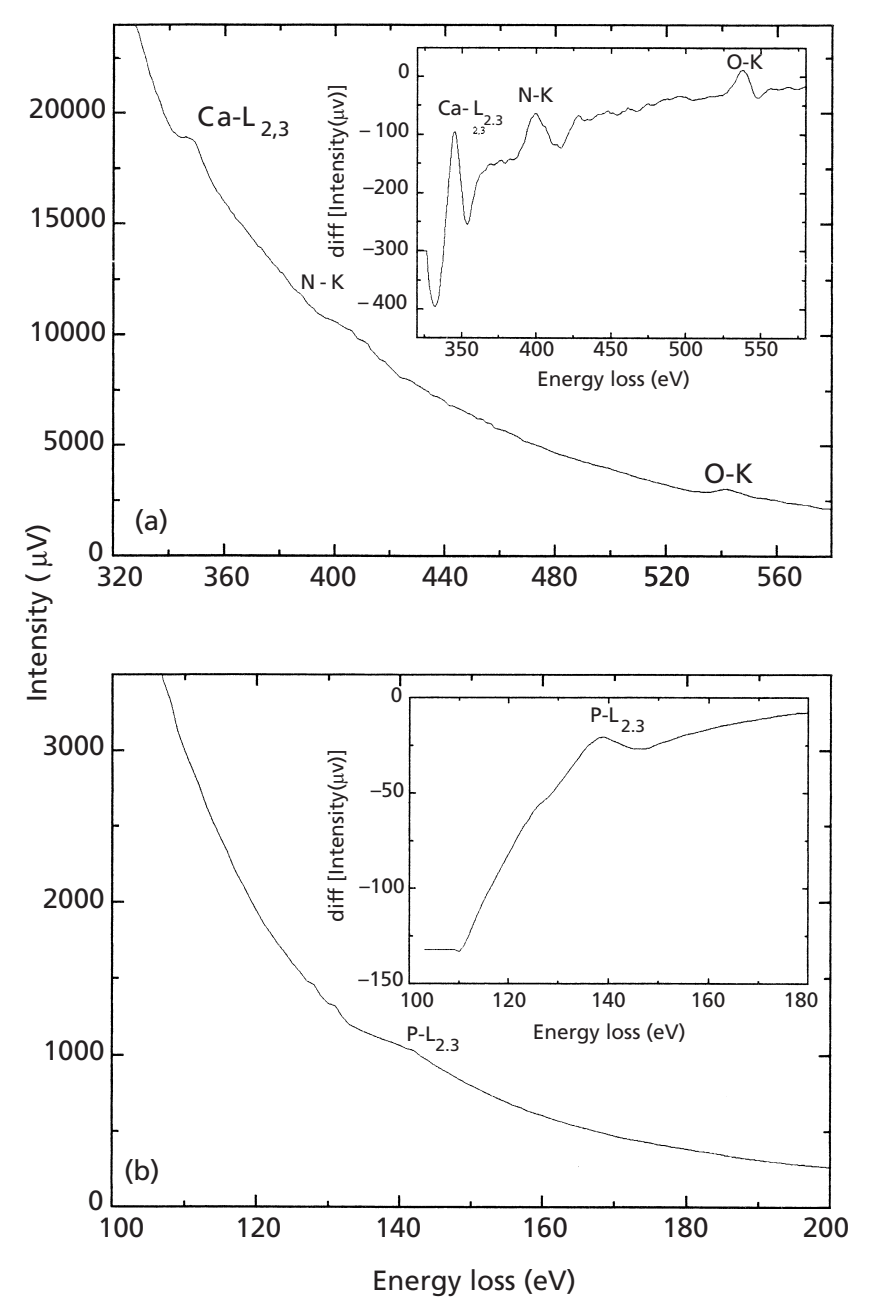

Fig. 5. Electron energy loss spectrum of an acidocalcisome showing (a) the $L_{2,3}$ edge of the element calcium ( $\left.346 \mathrm{eV}\right)$ and the $\mathrm{K}$ edges of the elements nitrogen $(402 \mathrm{eV})$ and oxygen $(532 \mathrm{eV})$, and (b) the $L_{2,3}$ edge of the element phosphorus $(132 \mathrm{eV})$. Insets are the first derivatives of the spectra shown, highlighting the edges of the elements. $K$ and $L_{2,3}$ are spectroscopic notation for the inner shells of the corresponding elements.

1998), a $\mathrm{Ca}^{2+}$-ATPase (Lu et al., 1998) and a pyrophosphatase (Scott et al., 1998) in the membrane of the acidocalcisome, identified as a membrane-bound organelle containing an electron-dense inclusion which occupies the whole organelle as seen in cryosections (Scott et al., 1997) or part of it as seen in transmission electron microscopy of routinely processed cells. The available data suggest that this organelle plays an important role in the homeostasis of $\mathrm{Ca}^{2+}$, whose mobilization was shown to influence host cell infection by $L$. amazonensis amastigotes (Lu et al., 1997). $\mathrm{Ca}^{2+}$ present in these organelles may also be involved in the stacking of parasite membranes, since it can promote a rapid interaction between multilamellar phospholipid vesicles (Rand et al., 1985), resembling the myelin-like figures observed in SBI-treated L. amazonensis.
Previous studies have shown that drugs such as acriflavine and suramin (Macadam \& Williamson, 1974a, b) also induce the appearance of electron-dense inclusion vesicles. However, further studies are necessary to determine whether they are of the same nature. Our observations on SBI-treated cells showed the presence of a large number of normal and polymorphic electron-dense acidocalcisome-like organelles surrounded by profiles of the ER. The images obtained were suggestive of an autophagic process, as characterized in detail in other cell systems (reviewed by Dunn, 1990, 1994). It is well known that drugs such as suramin (Macadam \& Williamson, 1974b) can induce autophagy but the mechanisms underlying this process remain to be clarified. ER breakdown and phospholipid accumulation have been reported in drug-treated T. rhodesiense (Macadam \& Williamson, 1974b). The phosphorus content of these organelles might be derived from rRNA autophagic hydrolysis since ribosome degradation has been reported (Macadam \& Williamson, 1974b). This seems unlikely in ketoconazole- and terbinafine-treated L. amazonensis since, contrary to membrane whorls, ribosome aggregates were not observed in forming autophagic vacuoles. It is reasonable to suppose that the enhanced membranous content of the autophagic vacuoles indicates an altered phospholipid turnover rate. The antifungal effects of SBI are believed to involve the accumulation of aberrant and toxic sterols in the plasma membrane (Groll et al., 1998) but cytoplasmic lipid deposits were also observed in SBI-treated Leishmania, possibly resulting from a dysfunction in autophagy regulation (Vannier-Santos et al., 1995).

Enzyme cytochemistry demonstrated the presence of reaction products indicative of acid phosphatase activity associated with some acidocalcisomes, thus suggesting their relation with lysosomes. Plasmodium acid phosphatase- and hydrolase-positive compartments, rather similar to the ones observed here, have been reported to be localized near digestive vacuoles in close contact with residual bodies (Slomianny \& Prensier, 1990). The cytochemical detection of hydrolytic enzymes does not necessarily imply their activity in these compartments under natural conditions, but the intergradation of the acidocalcisome contents strongly suggests a digestive function.

The detection of both fluid-phase and receptor-mediated endocytic tracers within acidocalcisome-like compartments further supports the association of these organelles with the endosomal/lysosomal pathway. Interestingly, we have previously observed that goldlabelled transferrin endocytosed by $L$. amazonensis amastigotes is delivered to lysosomes rather than recycled (Borges et al., 1998) as in African trypanosomes (Grab et al., 1992). The detection of nitrogen by elemental mapping may be indicative of the presence of proteins in these compartments. In this regard it is important to point out that the concentration of polyphosphate synthesized in mammalian cells is much higher than that of $\mathrm{P}_{\mathrm{i}}$ plus ATP (Kumble \& Kornberg, 1995). Therefore, the participation of membrane com- 
ponents is suggested (Kornberg, 1995). Interestingly both phosphate (Rand et al., 1985; Pisoni \& Lindley, 1992) and calcium (Haller et al., 1996; Kempler, 1985; Lemons \& Thoene, 1991) are incorporated into lysosomes of mammalian cells. The function of the phosphate environment in the autophagic pathway may be especially relevant in micro-organisms that accumulate polyphosphate. In yeast cells up to $99 \%$ of the polyphosphate is found in lysosome-related vacuoles (Kornberg, 1995). The lysosomal participation in the formation of the compartment is further supported by sialic acid detection using gold-labelled Limax flavus agglutinin (Vannier-Santos et al., 1991). It is noteworthy that pyrophosphate is the most abundant phosphate compound in trypanosomatid and apicomplexan parasites (Urbina et al., 1998).

Based upon the inability to accumulate gold-labelled transferrin (Scott et al., 1997), T. cruzi acidocalcisomes have been reported to not be involved in the endosomal pathway. Nevertheless, gold-labelled transferrin, unlike BSA, was only detected in a small part of the $T$. congolense endosomal system and therefore may not be a good endocytic tracer. It cannot be ruled out that among different species and under distinct experimental conditions these compartments may display discrete chemical compositions (Scott et al., 1997) and therefore play different roles. It has been pointed out that there is no evidence that Trypanosoma brucei acidocalcisomes are separate organelles and the acidic calcium pools may be related to lysosomes or endocytic vesicles (Xiong et al., 1997) as in mammalian cells. The detection of acid phosphatase and endocytic tracers in Leishmania $\mathrm{Ca}^{2+}$ and phosphorus-rich compartments strongly suggests that they may intersect lysosomes or residual bodies of the endocytic/autophagic pathway which are found in normal parasites but may be enhanced by chemotherapy-induced metabolic dysfunction. These observations suggest a close association of the endosomal/ lysosomal system with acidocalcisomes during the autophagic process in normal parasites or induced by antimicrobial agents in Leishmania.

\section{ACKNOWLEDGEMENTS}

The authors thank Dr D. P. Bazett-Jones from the Department of Anatomy and Medical Biochemistry, Faculty of Medicine, University of Calgary, Alberta, Canada, for helpful suggestions. This work was supported by Fundação de Amparo à Pesquisa do Estado do Rio de Janeiro (FAPERJ), Conselho Nacional de Desenvolvimento Científico e Tecnológico $(\mathrm{CNPq})$, Financiadora de Estudos e Projetos (FINEP), Fundação Universitária José Bonifácio (FUJB), Third World Academy of Sciences (TWAS), Conselho de Ensino para Graduados (CEPG-UFRJ) and Programa dos Núcleos de Excelência (PRONEX/MCT).

\section{REFERENCES}

Behamdan, K. A., Tallab, T. M., Johargi, H., Ibrahim, K., El Sherbini, A. H., Karkashan, E., Khare, A. K. \& Nauri, M. M. (1997). Terbinafine in the treatment of cutaneous leishmaniasis: a pilot study. Int J Dermatol 36, 59-60.
Berger, B. J. \& Fairlamb, A. H. (1992). Interactions between immunity and chemotherapy in the treatment of trypanosomiasis and leishmaniasis. Parasitology 105, S71-S78.

Berman, J. D. (1988). Chemotherapy for leishmaniasis: biochemical mechanisms, clinical efficacy and future strategies. Rev Infect Dis 10, 560-589.

Bird, R. G., Molloy, J. O. \& Ormerod, W. E. (1966). Granules and tubules in the cytoplasm of the sleeping sickness trypanosome: an electron microscope study. Trans $R$ Soc Trop Med Hyg 60, 753-760.

Borges, V. M., Vannier-Santos, M. A. \& De Souza, W. (1998). Subverted transferrin trafficking in Leishmania-infected macrophages. Parasitol Res 84, 811-822.

Carvalho, T. U. \& De Souza, W. (1977). Fine structure and X-ray microanalysis of electron-dense granules in Herpetomonas samuelpessoai. J Parasitol 63, 1116-1117.

Carvalho, T. U., Souto-Padón, T. \& De Souza, W. (1979). Herpetomonas samuelpessoai: electron microscopy and cytochemistry of electron-dense granules. Exp Parasitol 47, 297-304.

Chance, M. L. (1995). New developments in the chemotherapy of leishmaniasis. Ann Trop Med Parasitol 89, 37-43.

Dunn, W. A., Jr (1990). Studies on the mechanisms of autophagy: formation of the autophagic vacuole. J Cell Biol 110, 1923-1933.

Dunn, W. A., Jr (1994). Autophagy and related mechanisms of lysosome-mediated protein degradation. Trends Cell Biol 4, 139-143.

Dvorak, J. A., Engel, J. C., Leapmam, R. D., Swyt, C. R. \& Pella, P. A. (1988). Trypanosoma cruzi: elemental composition heterogeneity of cloned stocks. Mol Biochem Parasitol 31, 14-26.

Grab, D. J., Wells, C. W., Shaw, M. K., Webster, P. \& Russo, D. C. W. (1992). Endocytosed transferrin in African trypanosomes is delivered to lysosomes and may not be recycled. Eur J Cell Biol 59, 398-404.

Graham, R. C. \& Karnovsky, M. J. (1966). The early stages of absorption of injected horseradish peroxidase in the proximal tubules of mouse kidney: ultrastructure cytochemistry by a new technique. J Histochem Cytochem 14, 291-302.

Groll, A. H., De Lucca, A. J. \& Walsh, T. J. (1998). Emerging targets for the development of novel antifungal therapeutics. Trends Microbiol 6, 117-124.

Haller, T., Dietl, P. \& Deetjen, P. (1996). The lysosomal compartment as intracellular calcium store in MDCK cells: a possible involvement in $\mathrm{InsP}_{3}$-mediated $\mathrm{Ca}^{2+}$ release. Cell Calcium 19, $157-165$.

Herbert, I. V. (1965a). Cytochemistry of in vitro cultured Trypanosoma theileri. Exp Parasitol 16, 348-362.

Herbert, I. V. (1965b). Cytoplasmic inclusions and organelles of in vitro cultured Trypanosoma theileri and Trypanosoma melophagium and some speculations on their function. Exp Parasitol $17,24-40$.

Heywood, P., Weinman, D. \& Lipman, M. (1974). Fine structure of Trypanosoma cyclops in noncellular cultures. J Protozool 21, 232-238.

Janovy, J., Jr, Lee, K. W. \& Brumbaugh, J. A. (1974). The differentiation of Herpetomonas megaseliae: ultrastructural observations. J Protozool 21, 53-59.

Kempler, M. S. (1985). An adenosine triphosphate-dependent calcium uptake pump in human neutrophil lysosomes. J Clin Invest 76, 303-310.

Kornberg, A. (1995). Inorganic polyphosphate: toward making a forgotten polymer unforgettable. J Bacteriol 177, 491-496. 
Kumble, K. D. \& Kornberg, A. (1995). Inorganic polyphosphate in mammalian cells and tissues. J Biol Chem 270, 5818-5822.

Lemons, R. M. \& Thoene, J. G. (1991). Mediated calcium transport by isolated human fibroblast lysosomes. J Biol Chem 266, 14378-14382.

Lu, H.-G., Zhong, L., Chang, K.-P. \& Docampo, R. (1997). Intracellular $\mathrm{Ca}^{2+}$ pool content and signaling and expression of a calcium pump are linked to virulence in Leishmania mexicana amazonensis amastigotes. J Biol Chem 272, 9464-9473.

Lu, H.-G., Zhong, L., De Souza, W., Benchimol, M., Moreno, S. \& Docampo, R. (1998). $\mathrm{Ca}^{2+}$ content and expression of an acidocalcisomal calcium pump are elevated in intracellular forms of Trypanosoma cruzi. Mol Cell Biol 18, 2309-2323.

Macadam, R. F. \& Williamson, J. (1974a). Drug effects on the fine structure of Trypanosoma rhodesiense: acriflavine, ethidium and antrycide. Ann Trop Med Parasitol 68, 291-299.

Macadam, R. F. \& Williamson, J. (1974b). Drug effects on the fine structure of Trypanosoma rhodesiense: suramin, tryparsamide and mapharside. Ann Trop Med Parasitol 68, 301-306.

Olliaro, P. L. \& Bryceson, A. D. M. (1993). Practical progress and new drugs for changing patterns of leishmaniasis. Parasitol Today 9, 323-328.

Pisoni, R. L. \& Lindley, E. R. (1992). Incorporation of $\left[{ }^{32} \mathrm{P}\right]$ orthophosphate into long chains of inorganic polyphosphate within lysosomes of human fibroblasts. J Biol Chem 267, 3626-3631.

Rand, R. P., Kachar, B. \& Reese, T. S. (1985). Dynamic morphology of calcium-induced interactions between phosphatidylserine vesicles. Biophys J 47, 483-489.

Rangel, H., Dagger, F., Hernandez, A., Liendo, A. \& Urbina, J. A. (1996). Naturally azole-resistant Leishmania braziliensis promastigotes are rendered susceptible in the presence of terbinafine: comparative study with azole-susceptible Leishmania mexicana promastigotes. Antimicrob Agents Chemother 40, 2785-2791.

Reimer, L. (1991). Energy-filtering transmission electron microscopy. Adv Electron Phys 81, 43-126.

Robinson, J. M. \& Karnovsky, M. J. (1983). Ultrastructural localization of several phosphatases with cerium. J Histochem Cytochem 31,1197-1208.

Roth, J. (1983). The colloidal gold marker system for light and electron microscopic cytochemistry. In Techniques in Immunocytochemistry, Vol. 2, pp. 217-284. Edited by G. R. Bullock \& P. Petrusz. New York: Academic Press.

Scott, D. A. \& Docampo, R. (1998). Two types of $\mathrm{H}^{+}$-ATPases are involved in the acidification of internal compartments in Trypanosoma cruzi. Biochem J 15, 583-589.

Scott, D. A., Docampo, R., Dvorak, J. A., Shi, S. \& Leapman, R. D. (1997). In situ compositional analysis of acidocalcisomes in Trypanosoma cruzi. J Biol Chem 272, 28020-28029.

Scott, D. A., De Souza, W., Benchimol, M., Zhong, L., Lu, H. G., Moreno, S. \& Docampo, R. (1998). Presence of a plant-like proton- pumping pyrophosphatase in acidocalcisomes of Trypanosoma cruzi. J Biol Chem 273, 22151-22158.

Seed, J. R., Byram, J., III \& Gam, A. A. (1967). Characterization and localization of acid phosphatase activity of Trypanosoma gambiense. J Parasitol 14, 117-125.

Slomianny, C. \& Prensier, G. (1990). A cytochemical ultrastructural study of the lysosomal system of different species of malaria parasites. J Protozool 37, 465-470.

Urbina, J. A., Payares, G., Molina, J. \& 8 other authors (1996). Cure of short- and long-term experimental Chagas' disease using D0870. Science 273, 969-971.

Urbina, J. A., Moreno, B., Vierkotter, S., Oldfield, E., Moreno, S. N. J., Bailey, B. N., Yan, W., Scott, D. A. \& Docampo, R. (1998). Pyrophosphate is the most abundant high-energy phosphatecompound in trypanosomatid and apicomplexan parasites and a non-metabolizable analog. Mem Inst Oswaldo Cruz 93 (suppl. II), 72 .

Vannier-Santos, M. A., Saraiva, E. M. B. \& De Souza, W. (1991). Nuclear and cytoplasmic lectin binding sites in promastigotes of Leishmania. J Histochem Cytochem 39, 793-800.

Vannier-Santos, M. A., Urbina, J. A., Martiny, A., Neves, A. \& De Souza, W. (1995). Alterations induced by the antifungal compounds ketoconazole and terbinafine in Leishmania. J Eukaryot Microbiol 42, 337-346.

Vercesi, A. E., Moreno, S. J. N. \& Docampo, R. (1994). $\mathrm{Ca}^{2+} / \mathrm{H}^{+}$ exchange in acidic vacuoles of Trypanosoma brucei. Biochem J 304, 227-233.

Vickerman, K. \& Preston, T. M. (1976). Comparative cell biology of the kinetoplastid flagellates. In Biology of the Kinetoplastida, Vol. 1, pp. 35-130. Edited by W. H. R. Lumsden \& D. A. Evans. London: Academic Press.

Vickerman, K. \& Tetley, L. (1977). Recent ultrastructural studies on trypanosomes. Ann Soc Belge Med Trop 57, 441-455.

Watson, L. P. \& Lee, C. M. (1975). Ultrastructure of Trypanosoma duttoni. Z Parasitenkd 46, 133-140.

Werbovetz, K. A., Jeronimo, S. M. B., McDonald, T. L. \& Pearson, R. D. (1992). Treatment of leishmaniasis and trypanosomiasis. Curr Opin Infect Dis 5, 840-848.

Weinman, D. (1971). Malaysian primate trypanosomes: intracytoplasmic pigment. Southeast Asian J Trop Med Public Health $2,87$.

Williamson, J. \& McLaren, D. J. (1981). Localization of phosphatases in Trypanosoma rhodesiense. J Protozool 28, 460-467.

Xiong, Z. H., Ridgley, E. L., Enis, D., Olness, F. \& Ruben, L. (1997). Selective transfer of calcium from an acidic compartment to the mitochondrion of Trypanosoma brucei. I Biol Chem 272, 31022-31028.

Received 24 August 1998; revised 10 June 1999; accepted 6 July 1999. 